\title{
Atrial Septal Aneurysm during Pregnancy: A Case Report
}

\author{
Altraigey $\mathrm{A}^{1 *}$, Mosad $\mathrm{A}^{\mathbf{1}}$ and Tawfik $\mathrm{W}^{2}$ \\ ${ }^{1}$ Department of Obstetrics and Gynaecology, Benha \\ University, Benha, Egypt \\ ${ }^{2}$ Department of Cardiology, Benha University, Benha, \\ Egypt \\ *Corresponding author: Altraigey A, Department of \\ Obstetrics and Gynaecology, Benha University, Benha, \\ Egypt
}

Received: J une 19, 2018; Accepted: July 31, 2018;

Published: August 07, 2018

\section{Abstract}

Objective: Our objective was to endorse the management of women having atrial septal aneurysm in pregnancy. Background: Atrial Septal Aneurysm (ASA) is a rare cardiac defect that infrequently encountered during pregnancy, but its management should be optimized in order to avoid possible maternal morbidity.

Case Presentation: Hereby, we report a case of a woman in her early third trimester of pregnancy who presented with chest tightness and shortness of breath. On examination, there was irregular tachycardia and her echocardiography showed atrial septal aneurysm with mitral regurgitation. Multidisciplinary team management was initiated during pregnancy till uneventful delivery.

Conclusion: Regular follow-up with monthly echocardiography, control of arrhythmia and thromboprophylaxis were the corner stone for safe motherhood in such case.

Keywords: Atrial Aneurysm; Septal Aneurysm; Arrhythmia; Complicated Pregnancy

\section{Introduction}

Atrial Septal Aneurysm (ASA) is an uncommon but noteworthy cardiac defect of uncertain prevalence, which is mostly accompanied by congenital or acquired cardiac diseases. It may present as isolated totally asymptomatic condition [1]. Although ASA is mainly congenital, its clinical symptoms such as dyspnoea, palpitation, angina or thromboembolic accidents start during the second or third decades of patients' lives [2]. However, pregnancy is associated with a marked plasma volume expansion and cardiac output increase, which loads significantly the cardiovascular system. The physiologic adaptations of pregnant females to these hemodynamic loading include increasing both heart rate and stroke volume and fall in vascular resistance and blood pressure [3]. Diagnosis of ASA is disingenuous with pregnancy as its clinical picture is similar to a wide range of normal pregnancy complaints. There were no clear guidelines about management of such condition during pregnancy as well as the literature. Thus, we tried to highlight our experience with what seems to be a considerable cardiac disorder.

\section{Case Presentation}

19 years old primigravida presented at 28 weeks' gestation to her routine antenatal clinic appointment in Benha University Hospitals complaining of chest tightness and shortness of breath. Her pulse was irregular and reaching $130 \mathrm{bpm}$. Otherwise her other vital signs were stable. Abdominal examination revealed soft and lax gravid uterus with fundal height corresponding to her dates and audible foetal heartbeat. There was good perception of foetal kicks and no gush of fluid or vaginal bleeding. Her ultrasound showed single living healthy foetus with to date biometric and Doppler parameters.

The woman was referred immediately to emergency unit where she was reviewed by senior cardiologist. Initially, her electrocardiography was showing no abnormalities, but her echocardiography discovered the presence of non-mobile atrial septal aneurysm towards the right side without visible thrombosis or shunt at the trans-thoracic echocardiogram level. Her atrial dimension, ventricular dimensions and valves were normal and no segmental wall motion abnormalities could be detected at rest. Beta-blockers and aspirin were prescribed to the woman. There was a strong family history of cardiac lesions and she had a sister who died from a congenital heart defect.

The case was followed up on weekly outpatient basis as a highrisk case. Her echocardiography was repeated at monthly intervals. At 36 weeks' gestation, it showed extra findings as prolapsed anterior mitral valve leaflet with moderate to severe mitral regurgitation was detected with normal other valves. Also, her left ventricular dimensions were dilated with mild systolic dysfunction. The recommendation by cardiologist included that as long as she was haemodynamically stable and showed no signs of arrhythmias or thrombosis, she can go for spontaneous vaginal delivery or caesarean section according to obstetric decision with appropriate timing and dose of thromboprophylaxis. Aspirin was stopped at the same visit.

Senior anaesthetist carefully reviewed the case and decided that she is candidate for general or regional anaesthesia whenever needed and applicable. She was delivered by caesarean section at 39 weeks' gestation as she started to have labour pains and progressive cervical dilatation with breech presentation. Her postoperative follow-up was uneventful and she was discharged home on day three after the procedure. Thromboprophylaxis started 12 hours postoperative and continued till six weeks postpartum. After puerperium she started to follow up with cardiology clinic.

\section{Discussion}

In 1934, Lang and Posselt were the first to report case of ASA [1]. ASA is generally a localized defect which involves the interatrial septum and occurs at the fossa ovalis level [4], but rarely it might involve the entire septum bulging towards to the left, right atrium or both. ASA, either by association or as possible aetiology, is related to 


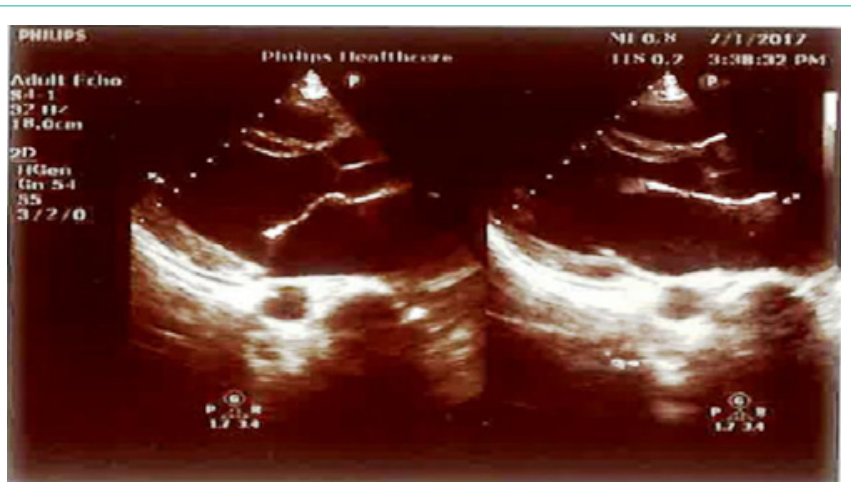

Figure 1: Echocardiography' parasternal long axis view (PLAX) shows prolapsed anterior mitral valve leaflet.
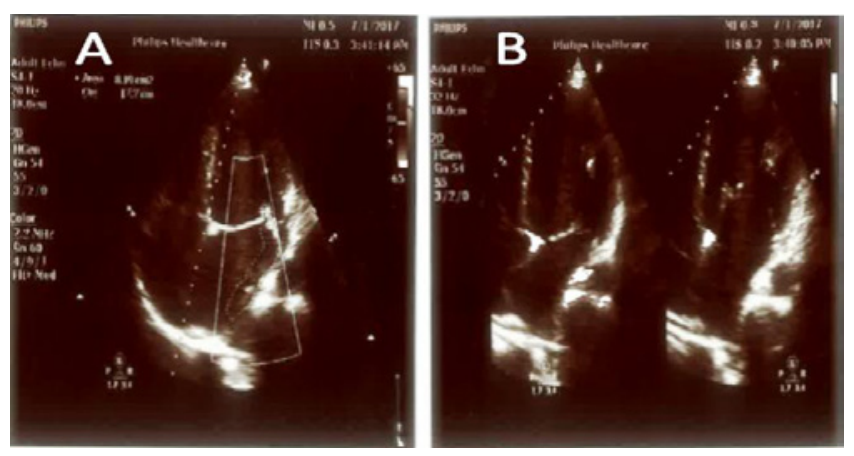

Figure 2: Echocardiography' apical four-chamber view shows moderate eccentric posteriorly directed mitral regurgitation $(A)$ and redundant intraatrial septum with atrial septal aneurysm (B).

atrial septal defect, valve prolapse, systolic clicks, atrial arrhythmias, systemic and pulmonary embolism [5]. In one large study, Hanley et al. stated that ASA could be clearly diagnosed by the non-invasive two-dimensional echocardiography and the observer should search for other associated defects [6]. Other imaging modalities included Computerized Topography (CT) especially for the large aneurysms [2]. ASA increased the risks of maternal morbidities as atrial arrhythmias, systemic embolism, and myocardial dysfunction up to heart failure. Thus, surgical treatment might be essential to prevent complications [2]. However, conservative management could be implemented provided that there were no other mechanical defects as atrial septal defect or proved thromboembolic incidents especially during pregnancy $[2,7]$.

Drug administration as beta-blockers, aspirin and anticoagulants might be an option for ASA cases with mild symptoms. Although conservative strategy increased the risk of morbidity, cardiac surgeries had its own challenges particularly during pregnancy. Using medical management necessitated monitoring the changes in the ASA, left atrial and ventricular dimensions as well as their systolic functions because dilation of the ASA could trigger atrial tachyarrhythmia or compress nearby cardiac structures as the coronary arteries or ventricular walls [8]. In a recent systematic review authors concluded that aneurysm size did not predict thrombus formation and embolic events, in contrast to atrial tachyarrhythmia [9]. Therefore, clinical observation of symptoms was also essential. To date, approximately fifty ASA case reports were reported in the literature. To our knowledge, this is the first to address the management throughout pregnancy, labour and delivery.

\section{Author Contribution}

Authors contributed to the case report design, shared in data collection, wrote the first manuscript draft. Authors shared equally in the critical appraisal of the manuscript, fully agreed to its consistency and accuracy. They approved the final manuscript. Ahmed Altraigey guarantees the manuscript's accuracy and the contributor-ship.

\section{Patient Consent}

The patient as well as her husband was consented to use her data scientifically without any burden on her confidentiality.

\section{References}

1. Jatav RK, Kumbhare MB, Surender T, Rachan Ch, Krishna TVV. Atrial septal aneurysm in adult patients: spectrum of clinical, echocardiographic presentation and to propose a new classification on the basis of transthoracic-two-dimensional echocardiography. Int J Res Med Sci. 2014; 2: 708717.

2. Chen $Y$, Mou $Y$, Jiang LJ, Hu SJ. Congenital giant left atrial appendage aneurysm: a case report. J Cardiothorac Surg. 2017; 12: 15.

3. Clapp JF, Capeless E. Cardiovascular function before, during and after the first and subsequent pregnancies. Am J Cardiol. 1997; 80: 1469-1473.

4. Oyedeji AT, Okunola O, Sani MU. Atrial Septal Aneurysm Mimicking a Cor Triatriatum Sinister: A Case Report and Review of the Literature. Clin Med Insights Case Rep. 2012; 5: 143-147.

5. Kariyanna PT, Jayarangaiah A, Adrah R, Sharma A. Huge Interatrial Septum Aneurysm. American Journal of Medical Case Reports. 2016; 4: 1111-1114.

6. Hanley PC, Tajik AJ, Hynes JK, Edwards WD, Reeder GS, Hagler DJ, et al. Diagnosis and Classification of Atrial Septal Aneurysm by Two-Dimensional Echocardiography: Report of 80 Consecutive Cases. J Am Coll Cardiol. 1985; 6: 1370-1382.

7. Wang $H$, Lei F, Yang $Y$, Ruan F, Sun $X$, Sun J, et al. Clinical experience of intraoperative device closure of interatrial septal aneurysm with atrial septal defects. SAS J. Surg. 2016; 2: 142-145.

8. Cujec B, Bharadwaj B, Orchard RC, Lopez JF. Transesophageal echocardiography in the diagnosis of left atrial appendage aneurysm. J Am Soc Echocardiogr. 1990; 3: 408-411.
Austin J Obstet Gynecol - Volume 5 Issue 7 - 2018 Submit your Manuscript | www.austinpublishinggroup.com Altraigey et al. (@) All rights are reserved
Citation: Altraigey A, Mosad A and Tawfik W. Atrial Septal Aneurysm during Pregnancy: A Case Report. Austin J Obstet Gynecol. 2018; 5(7): 1119. 